\title{
The Impact of Video Feedback on Acquiring Competency in Basic Surgical Skills (Sterile Working) in an Undergraduate Medical Training Program: A Comparative Effectiveness Analysis
}

\author{
Moritz Muessig ${ }^{1}$, Jasmina Sterz ${ }^{1}$, Maria-Christina Stefanescu², Bernd Bender ${ }^{1}$, Sebastian H. Hoefer ${ }^{2}$, \\ Miriam Ruesseler ${ }^{1}$ \\ ${ }^{1}$ Department of Trauma, Hand and Reconstructive Surgery, University Hospital Frankfurt, Germany \\ ${ }^{2}$ Department of Surgery, University Hospital Frankfurt, Germany \\ Email: miriam.ruesseler@kgu.de
}

\begin{abstract}
Feedback is an essential part of the learning process during undergraduate medical training especially in acquiring practical skills. This study investigates the impact of video feedback on the acquisition of sterile working competencies as an example of a complex practical skill. Fourthyear medical students enrolled in the mandatory skills training completed a 210-minute training unit of ,wound care'. Oral feedback was received directly after the practical procedure. In the study group, the performance was video-taped and reviewed directly thereafter. Afterwards, students completed a 5-minute OSCE. One to three month later, students were re-assessed as part of their regular practical skills assessment. A total of 107 students were included in the study. There were no significant differences between the two study groups at both point in times. In the present setting and skill, video feedback seems to have no impact on skills' acquisition.
\end{abstract}

Key Words: Feedback, practical skills, video feedback, medical training

\section{Introduction}

Feedback is recognised as an essential component in medical education [1,2]. Clinical feedback is defined as 'specific information about the comparison between a trainee's observed performance and a standard, given with the intent to improve the trainee's performance' [2]. What is subsumed under the term 'feedback' can take many forms - feedback can be delivered during or after a procedure $[3,4]$, it can be delivered by peers or experts, individually or in groups, and it can be delivered using a multitude of techniques including oral, written, or various types of video feedback [5-7].

In addition to solely verbal feedback, reviewing one's performance by video may be a useful adjunct to the debriefing process. The existing experimental outcomes comparing video feedback to verbal feedback are mixed [8]. For instance, video feedback has been used highly successfully in training anaesthesiology residents perform epidural anaesthesia [9], in emergency room simulations [10, 11], in the acquisition of wound suturing skills by medical students [12], during a simulated patient encounter in surgical context [13], and in the acquisition of laparoscopic surgical skills by surgery residents [14]. Other studies, found that in some settings, this technique is as effective, but not more so, as traditional types of feedback, e.g. Byrne et al. [15] in a simulation of general anaesthesia. When video feedback was used in a simulated emergency training for anaesthetists focussing on non-technical skills, those participants who had been given feedback using video recordings actually scored lower on the exclusively non-technical skills that were the focus of this study, than those who had received oral feedback [16]. Overall, the available literature suggests that video feedback in medical training seems to be more useful in the acquisition of technical, practical skills - such as those found in surgical disciplines, than in non-technical skills.

One of the most fundamental competencies in surgery is the hygienically perfect performance. Hospital-acquired infections remain a substantial problem even in highly developed medical systems [17]. Sterile working requires manual dexterity, awareness of oneself, one's own actions and one's surroundings, knowledge of when, and how, breaches in sterility occur, as well as personal integrity with 
regards to reporting breaches in sterility in order to ensure that such breaches are minimised in the first place, and, when they do occur, are noticed and acknowledged reliably.

In order to teach sterile work before students actually start their rotations and enter the operating room, simulation training is an established method offering a safe environment without putting patients at risk [18].

The aim of the present study was to analyse the impact of video feedback versus oral feedback on acquiring competencies in sterile working directly after feedback and in the long term.

\section{Methods}

\subsection{Trial Design}

The study presents a comparative randomized effectiveness analysis with two parallel study arms aiming to analyse the impact of video feedback versus oral feedback on acquiring competencies in sterile working.

The study was conducted according to ethical principles of the Helsinki Declaration (Ethical Principles for Medical Research Involving Human Subjects). According to the Ethics Board at Goethe University Medical School, no Ethics Approval was required for conducting this study.

\subsection{Participants}

Study participants were fourth-year medical students who were enrolled in the mandatory rotation in surgery in the winter semester of 2015/16 as part of the six-year medical undergraduate curriculum. In their third year, the year before enrolling in the rotation in surgery, students had had the opportunity to attend two lecture series called 'Introduction to Surgery' and 'Main Lectures in Surgery'. Whilst students' attendance at these lectures was optional, both lecture series were followed by mandatory multiple choice examinations which to pass was a precondition to being admitted to the rotation.

\subsection{Study Protocol}

The rotation in surgery lasts three weeks in total and is offered four times during the semester. Each three-week rotation begins with a one-week skills lab training called 'Training of Practical Skills'[19]. At the beginning of each rotation, students received comprehensive information regarding the study, its goals, and the intended use of participants' data. Participation was voluntary and volunteers were asked to sign written Statements of Consent. Students were blinded towards their later affiliation to a particular study group. Basic data regarding, for example, students' age, sex and duration of studies up to the beginning of the study were collected with a questionnaire.

Prior to the training week - independent of the authors and independent of study participation - all students who completed their surgical training within the study period were assigned by the Frankfurt Medical School's Dean's Office to one of the learning groups per training week (with a maximum of 6 students per group). These learning groups were randomly assigned to either video feedback or oral feedback.

During the one-week skills lab training, students rotate through 12 training modules covering basic surgical skills such as wound care, suturing, inserting an intravenous line, or how to behave in an operating room. In addition these technical skills, strong emphasis was being put on teaching communication skills and proper behaviour and conduct towards the patient. At the end of the semester, all students have to successfully pass a 10-station OSCE.

The study itself was conducted as part of the teaching module 'wound care', a module which is held by specifically trained and experienced student peer tutors. During the module, students learn how to correctly perform wound management. Learning objectives for the module are defined as correct sterile working during the whole performance, which includes initially cleaning the wound, then preparing a sterile table/working surface for preparing sterile supplies for the later suturing of the wound, putting on sterile gloves, covering the wound with a sterile adhesive aperture drape, performing local anaesthesia and finally performing a simple interrupted suture. Competencies in these learning objectives are 
acquired in a stepwise manner under the demonstration and supervision of the peer tutor. In the simulated environment, authentic hospital supplies are used while the required scientific and clinical background is presented to students using a standardised slide presentation.

\subsection{Intervention}

As part of the module, students have to demonstrate the procedure under supervision of the peer tutor. In the Oral Feedback group, two students at a time performed the exercise whilst being observed by peer tutors taking notes. Feedback given to students who had been assigned to the Oral Feedback group was given based on the ALOBA guidelines for giving feedback, meaning that an 'agenda-led, outcomebased' structure was employed where participants were encouraged to reflect themselves on their performance and how it could be improved [20]. The process of giving oral feedback was moderated by the peer tutor and began with the students who were to be given feedback initially reflecting themselves on their performance and potential for improvement, which was then followed by feedback from the student partner. Only in the end did the peer tutor conclude the feedback round with constructive commentary based on the notes taken during the exercise and a summary of the students' strengths and weaknesses.

In the Video Feedback group, pairs of students were videotaped using Sony Camcorders (Sony Corp., Tokyo, Japan). The videos were reviewed immediately after the performance, again based on the ALOBA 'agenda-led, outcome-based' structure. The subsequent feedback was performed as described for the oral feedback group. For reasons of privacy and data protection, the videotapes were deleted immediately after review.

After both oral and video feedback, all students had the opportunity to exercise again and to improve on the areas mentioned in the feedback.

\subsection{Measurement}

To assess acquired competencies, the objective structured clinical examination (OSCE) format was used, a valid and reliable assessment instrument for clinical competence [21, 22]. The first measurement (here referred to as Point in Time One) took place either on the same day or in the morning of the day after the initial exercise. Students had to complete a 5-minute OSCE station similar to what they had trained earlier in the module 'wound management'. The second measurement took place at the end of the semester as a part of a mandatory summative assessment. Sterile procedures were a key objective and were thus used as a point of measurement for the present study. The first station used for this study required students to set up a sterile working area with a full set of sterile instruments and supplies needed for later suturing, then putting on sterile gloves, and finally to correctly clean and disinfect the simulated wound without compromising sterility (the suturing itself was not a required task, as the focus of the present study was on hygienic and sterile working). In the second station, participants were tasked with preparing a simulated venous access port for administration of medication, requiring them to prepare a sterile working area, unpack all necessary material and equipment without compromising sterility, put on sterile gloves, disinfect the skin above the subcutaneous port, and insert the needle such that again sterility was not compromised.

At both point in time, directly after the initial skills lab training as well as during the OSCE at the end of the semester, students were rated on a checklist containing two parts for technical and nontechnical skills. For the procedural items assessing technical skills (Part A of the checklist), a trinary scoring system was used such that 0 points were given for 'not attempted, 1 point was given for 'attempted, but not finished correctly', and 2 points were given for 'finished correctly'. Non-technical skills, of which there were 6 items, were assessed on a 5 -point scale from ' 1 ' (unsatisfactory) to ' 5 ' (excellent), with precisely worded definitions for grades 1,3 , and 5 .

Examiners were blinded to the study group. They received detailed examiner training prior to the OSCE so that experience in the use of the checklist could be gained.

\subsection{Data Analysis}

The results were statistically analysed using the software SPSS (IBM Corp., Armonk, New York, USA). 
The data were statistically tested for normal distribution using the Kolmogorov-Smirnov-Test and, where data were thus found to be normally distributed, the differences in the scores between the study groups were analysed using Student's t-tests for independent samples and are presented as mean \pm standard deviation. Where data were not found to be normally distributed, it was further analysed using the Kolmogorov-Smirnov-Test for independent samples. Significance levels were set to p $<0.05$.

\section{$3 \quad$ Results}

Of the students who completed their surgical training during the study period, 107 consented to participate in the study. Table 1 shows the socio-demographic data of the study participants. There were no significant differences between the two study groups. At Point in Time 1, there were no significant differences in the rated performance between both study groups, neither as pertains to technical skills (the checklist rating), nor in non-technical skills or the global rating (Tab 2a, b). At Point in Time 2, at the station 'wound management', 39 students had to be excluded from the study due to changed and thus no longer comparable exam conditions. At the station 'accessing a port-a-cath', all study participants could be analysed (Table 1). There were no statistically significant differences between the study groups regarding technical and non-technical skills, neither for performing simulated wound management (Tab. 3a, b), nor regarding the hygienic placement of a venous access port needle in a simulated port (Tab. $4 \mathbf{a}, \mathbf{b})$.

\section{Discussion}

Both performance reviews and appropriate, actionable feedback have been described as critical components of learning in general, and of medical education in particular [1, 7]. When learners receive feedback that is individualised and well-structured, the probability of correct performance is improved [18]. In the present study, we evaluate the feasibility and benefit of video feedback in undergraduate surgical education, finding that whilst video feedback was viable and practicable within our curricular constraints, no additional benefit over purely oral feedback could be established. Reviewing the existing literature on the subject reveals heterogeneous approaches and outcomes - performance reviews and feedback processes can be varied in how they are conducted in practice, and the experimental outcomes are mixed [8]. This suggests that the precise details of how performance reviews and feedback are conducted as well as the precise moment in a learner's educational development at which these techniques are employed play an important role in determining their effectiveness.

A major aspect of assessing a teaching technique is whether short or long term memory retention is investigated. In this study, the effect of video feedback on learning outcomes was measured first on the same or the day immediately following the learning session (short term retention), and then again several months later at the end of the semester (long term retention). Whilst previous studies suggest that benefits from video feedback improve when evaluated against long term memory retention, no such effect could be established in the present study [9]. In some other previous studies, this effect seems to have been particularly noticeable where participants had the chance to take video recordings at home and review them repeatedly [12], or get more accustomed to the video feedback process, potentially 'learning' how to derive the maximum benefit from video feedback [11].

Whilst the present study did not find an additional benefit of video feedback over purely oral feedback, important insights regarding video feedback techniques were gained. The trial described here assessed the effectiveness of video feedback in the context of a published educational concept, the 'Training of Practical Skills' [19], that is well established at the medical school where the trial was held. Our trial demonstrates that video feedback can be successfully integrated into a pre-existing, tightly structured training and that practical challenges that presented themselves could readily be overcome.

Such practical challenges lay in particular in the resource and time intensiveness of video feedback. In order to implement video feedback, additional technical equipment such as camcorders, laptops, spare batteries, spare memory cards, and tripods was acquired. The skills lab premises were adapted and rearranged such that two participants performing the simulated exercise simultaneously could be taped at the same time without interfering with each other or the training course in general and such that useand meaningful video material was obtained. Student peer tutors were trained in conducting the trial 
exercise, and additional staff was necessary to review videotapes with study participants and oversee video feedback sessions.

Specifically, reviewing videotapes proved to be more time intensive than purely oral feedback even when two groups of study participants engaged in feedback rounds in two separate rooms simultaneously. Whilst purely oral feedback on average took about 20 to 30 minutes to administer, video feedback, on average, took 40 to 60 minutes. This was due to the fact that for video feedback, the taped exercise was first replayed in its entirety and often, several scenes of particular interest were again replayed during the following feedback round/discussion. Thus, given the curricular schedule, those study participants who received video-based feedback had, on average, less time to exercise again after the feedback round compared to those participants who received oral feedback, suggesting that the benefit of adding video feedback to exhaustive training might be understated in the results presented here.

Our study has some limitations. Since it was conducted at a single medical school, on a single cohort of medical students, its explanatory power and transferability to other medical schools might be restricted; however, the sample size of 107 at the first measurement and 75 at the second, long term measurement, is relatively large when viewed in the context of the available literature.

Our study is distinct regarding the fact that its results were obtained from a real-life, 'in vivo', rather than 'in vitro', experimental setting. This means that it captures all real-life influences such as curricular constraints, changing tutors, or varying motivation of participating students. However, this also implies that the study design had to be adapted to fit those constraints, in particular as pertains to schedules, group sizes, the selection of participants (who had to be recruited from the third-year student cohort) and the randomisation of participants, who could only be randomised as whole groups, groups which were in turn determined by the Dean's Office prior to the commencement of this study.

Further, since the study was conducted over an entire semester it is conceivable that students at the later training courses may have gotten information on the exercise itself and/or on the learning objectives as defined in the checklist used at the final OSCE at the end of the semester. However, the fact that student performance did not significantly change (improve) towards the later training weeks suggests that this effect should be limited.

The strengths of this study include the relatively large sample size, the measurement of both short and long term memory retention, the real life setting, specifically trained tutors, a defined feedback structure, as well as trained and blinded OSCE examiners. In the design of further studies on video feedback, it might be taken into consideration whether it might be beneficial to allow participants to take video recordings home for repeated viewing, and whether students might derive the most benefit from video feedback when repeatedly using this technique in order to become best acquainted with its usefulness.

Acknowledgments. This study was funded by the German Federal Ministry of Education and Research (grant 01PL12038A) as part of the joint research project, "Practical clinical competence-a joint program to improve training in surgery." All authors declare that they have no further conflict of interest.

\section{References}

1. M. Eraut, "Feedback," Learning in Health and Social Care, vol. 5, pp. 111-118, 2006.

2. J. M. van de Ridder, K. M. Stokking, W. C. McGaghie et al., "What is feedback in clinical education?," Med Educ, vol. 42, pp. 189-197, 2008.

3. S. Parmar, C. P. Delaney, "The role of proximate feedback in skills training," Surgeon, vol. 9 Suppl 1, pp. S26 27, 2011.

4. J. N. Van Heukelom, T. Begaz, R. Treat, "Comparison of postsimulation debriefing versus in-simulation debriefing in medical simulation," Simul Healthc, vol. 5, pp. 91-97, 2010.

5. W. F. Bond, L. M. Deitrick, D. C. Arnold et al., "Using simulation to instruct emergency medicine residents in cognitive forcing strategies," Acad Med, vol. 79, pp. 438-446, 2004.

6. D. Raemer, M. Anderson, A. Cheng et al., "Research regarding debriefing as part of the learning process," Simul Healthc, vol. 6 Suppl, pp. S52-57, 2011. 
7. R. M. Fanning, D. M. Gaba, "The role of debriefing in simulation-based learning," Simul Healthc, vol. 2, pp. 115-125, 2007.

8. T. Levett-Jones, S. Lapkin, "A systematic review of the effectiveness of simulation debriefing in health professional education," Nurse Educ Today, vol. 34, pp. e58-63, 2014.

9. D. J. Birnbach, A. C. Santos, R. A. Bourlier et al., "The effectiveness of video technology as an adjunct to teach and evaluate epidural anesthesia performance skills," Anesthesiology, vol. 96, pp. 5-9, 2002.

10.S. Boet, M. D. Bould, B. Sharma et al., "Within-team debriefing versus instructor-led debriefing for simulationbased education: a randomized controlled trial," Ann Surg, vol. 258, pp. 53-58, 2013.

11.L. A. Scherer, M. C. Chang, J. W. Meredith et al., "Videotape review leads to rapid and sustained learning," Am J Surg, vol. 185, pp. 516-520, 2003.

12.A. L. Farquharson, A. C. Cresswell, J. D. Beard et al., "Randomized trial of the effect of video feedback on the acquisition of surgical skills," Br J Surg, vol. 100, pp. 1448-1453, 2013.

13.M. Ruesseler, J. Sterz, B. Bender et al., "The effect of video-assisted oral feedback versus oral feedback on surgical communicative competences in undergraduate training," Eur J Trauma Emerg Surg, vol. 2016.

14.G. G. Hamad, M. T. Brown, J. A. Clavijo-Alvarez, "Postoperative video debriefing reduces technical errors in laparoscopic surgery," Am J Surg, vol. 194, pp. 110-114, 2007.

15.A. J. Byrne, A. J. Sellen, J. G. Jones et al., "Effect of videotape feedback on anaesthetists' performance while managing simulated anaesthetic crises: a multicentre study," Anaesthesia, vol. 57, pp. 176-179, 2002.

16.G. L. Savoldelli, V. N. Naik, J. Park et al., "Value of debriefing during simulated crisis management: oral versus video-assisted oral feedback," Anesthesiology, vol. 105, pp. 279-285, 2006.

17.T. A. Brennan, L. L. Leape, N. M. Laird et al., "Incidence of adverse events and negligence in hospitalized patients: results of the Harvard Medical Practice Study I. 1991," Qual Saf Health Care, vol. 13, pp. 145-151; discussion 151-142, 2004.

18.S. B. Issenberg, W. C. McGaghie, E. R. Petrusa et al., "Features and uses of high-fidelity medical simulations that lead to effective learning: a BEME systematic review," Med Teach, vol. 27, pp. 10-28, 2005.

19.M. Russeler, R. Weber, A. Braunbeck et al., "[Training of practical clinical skills in surgery - a training concept for medical students]," Zentralbl Chir, vol. 135, pp. 249-256, 2010.

20.S. Kurtz, J. Silverman, J. Draper. Teaching and learning communication skills in medicine. CRC press; 2016.

21.B. Hodges, "Validity and the OSCE," Med Teach, vol. 25, pp. 250-254, 2003.

22.G. Regehr, H. MacRae, R. K. Reznick et al., "Comparing the psychometric properties of checklists and global rating scales for assessing performance on an OSCE-format examination," Acad Med, vol. 73, pp. 993-997, 1998.

Table 1. Characteristics of the study groups

\begin{tabular}{|c|c|c|c|c|c|c|}
\hline \multirow[b]{2}{*}{ Study Group } & \multicolumn{2}{|c|}{ Point in time 1} & \multicolumn{2}{|c|}{$\begin{array}{c}\text { Point in time } 2- \\
\text { Wound Management }\end{array}$} & \multicolumn{2}{|c|}{$\begin{array}{c}\text { Point in time } 2- \\
\text { Venous Access Port }\end{array}$} \\
\hline & $\begin{array}{c}\text { Oral } \\
\text { Feedback } \\
\end{array}$ & $\begin{array}{c}\text { Video } \\
\text { Feedback } \\
\end{array}$ & $\begin{array}{c}\text { Oral } \\
\text { Feedback } \\
\end{array}$ & $\begin{array}{c}\text { Video } \\
\text { Feedback } \\
\end{array}$ & $\begin{array}{c}\text { Oral } \\
\text { Feedback } \\
\end{array}$ & $\begin{array}{c}\text { Video } \\
\text { Feedback }\end{array}$ \\
\hline Number of participants & 53 & 54 & 39 & 36 & 53 & 54 \\
\hline Male & $\begin{array}{c}19 \\
(35.8 \%) \\
\end{array}$ & $\begin{array}{c}19 \\
(35.2 \%) \\
\end{array}$ & $\begin{array}{c}14 \\
(35.9 \%) \\
\end{array}$ & $\begin{array}{c}13 \\
(36.1 \%) \\
\end{array}$ & $\begin{array}{c}19 \\
(35.8 \%) \\
\end{array}$ & $\begin{array}{c}19 \\
(35.2 \%) \\
\end{array}$ \\
\hline Age (years) & 23.8 & 24.1 & 24.0 & 23.7 & 23.8 & 24.1 \\
\hline Duration of study (years) & 3.4 & 3.3 & 3.4 & 3.3 & 3.4 & 3.3 \\
\hline Number of previous OSCEs & 1 & 1 & 1 & 1 & 1 & 1 \\
\hline $\begin{array}{l}\text { Number of previous clinical } \\
\text { electives }\end{array}$ & 1.2 & 1.1 & 1.4 & 1.1 & 1.2 & 1.1 \\
\hline $\begin{array}{l}\text { Professional qualification as } \\
\text { - paramedic } \\
\end{array}$ & 3 & 4 & 2 & 2 & 3 & 4 \\
\hline - nurse & 5 & 4 & 2 & 3 & 5 & 4 \\
\hline
\end{tabular}


Table 2a. Point in Time 1: Performance results for technical skills for sterile working during wound management.

\begin{tabular}{l|l|l|l|l}
\hline & Oral Feedback & Video Feedback & p & d \\
\hline Overall score & $51.8 \% \pm 18.93$ & $56.1 \% \pm 19.5$ & 0.250 & 0.224 \\
\hline Preparation of hygienic working & $63.8 \% \pm 31.0$ & $60.8 \% \pm 30.1$ & 0.845 & 0.100 \\
\hline Preparation of sterile material & $79.3 \% \pm 18.3$ & $82.7 \% \pm 15.6$ & 0.910 & 0.205 \\
\hline Donning sterile gloves & $58.5 \% \pm 27.7$ & $64.4 \% \pm 28.6$ & 0.912 & 0.208 \\
\hline Wound disinfection & $32.6 \% \pm 39.9$ & $40.2 \% \pm 38.6$ & 0.373 & 0.191 \\
\hline Covering of wound with sterile drape & $15.8 \% \pm 30.8$ & $20.4 \% \pm 37.8$ & 0.979 & 0.132 \\
\hline
\end{tabular}

Data are presented as mean + standard deviation in \%. $\mathrm{p}=$ significance, $\mathrm{d}=$ Cohen's $\mathrm{d}$ (educational effect size).

Table 2b. Point in Time 1: Performance results for non-technical skills for sterile working during wound management

\begin{tabular}{l|l|l|l|l}
\hline & Oral Feedback & Video Feedback & $\mathrm{p}$ & $\mathrm{d}$ \\
\hline Overall score & $65.2 \% \pm 15.5$ & $64.5 \% \pm 18.2$ & 0.829 & 0.042 \\
\hline Introduction to the patient & $78.5 \% \pm 34.6$ & $71.1 \% \pm 37.2$ & 0.805 & 0.205 \\
\hline Articulation/ terminology & $77.4 \% \pm 23.6$ & $78.5 \% \pm 25.4$ & 0.995 & 0.047 \\
\hline Structure of working & $69.8 \% \pm 22.1$ & $69.6 \% \pm 26.8$ & 0.898 & 0.007 \\
\hline Behavior/professional attire and appearance & $65.3 \% \pm 23.3$ & $68.2 \% \pm 25.9$ & 0.916 & 0.117 \\
\hline Patient handling & $72.8 \% \pm 19.3$ & $71.1 \% \pm 26.3$ & 0.898 & 0.075 \\
\hline Management of working environment & $27.6 \% \pm 19.3$ & $28.2 \% \pm 19.6$ & 1.000 & 0.031 \\
\hline
\end{tabular}

Data are presented as mean + standard deviation in \%. $\mathrm{p}=$ significance, $\mathrm{d}=$ Cohen's $\mathrm{d}$ (educational effect size).

Table 3a. Point in Time 2: Performance results for technical skills for sterile working during wound management

\begin{tabular}{l|l|l|l|l}
\hline & Oral Feedback & Video Feedback & p & d \\
\hline Overall & $69.5 \% \pm 25.9$ & $68.4 \% \pm 30.2$ & 0.838 & 0.037 \\
\hline Preparation of hygienic working & $61.6 \% \pm 24.2$ & $69.8 \% \pm 26.3$ & 0.433 & 0.326 \\
\hline Preparation of sterile material & $74.5 \% \pm 24.8$ & $80.4 \% \pm 22.4$ & 0.687 & 0.247 \\
\hline Donning sterile gloves & $93.1 \% \pm 15.4$ & $85.2 \% \pm 28.3$ & 0.993 & 0.346 \\
\hline Wound disinfection & $80.2 \% \pm 29.5$ & $77.5 \% \pm 31.0$ & 1.000 & 0.088 \\
\hline Covering wound with sterile drape & $68.4 \% \pm 38.1$ & $69.5 \% \pm 40.0$ & 1.000 & 0.029 \\
\hline
\end{tabular}

Data are presented as mean + standard deviation in \%. $\mathrm{p}=$ significance, $\mathrm{d}=$ Cohen's d (educational effect size).

Table 3b. Point in Time 2: Performance results for non-technical skills for sterile working during wound management

\begin{tabular}{l|l|l|l|l}
\hline & Oral Feedback & Video Feedback & $\mathbf{p}$ & $\mathbf{d}$ \\
\hline Overall score & $75.2 \% \pm 25.9$ & $74.3 \% \pm 30.3$ & 0.936 & 0.034 \\
\hline Introduction to the patient & $90.0 \% \pm 16.2$ & $93.8 \% \pm 11.9$ & 0.899 & 0.264 \\
\hline Articulation/ terminology & $82.8 \% \pm 17.3$ & $88.1 \% \pm 18.2$ & 0.476 & 0.301 \\
\hline Structure of working & $71.7 \% \pm 16.8$ & $75.6 \% \pm 20.8$ & 0.687 & 0.209 \\
\hline Behavior/professional attire and appearance & $73.3 \% \pm 17.9$ & $75.0 \% \pm 22.1$ & 0.998 & 0.083 \\
\hline Patient handling & $87.2 \% \pm 20.4$ & $85.0 \% \pm 22.1$ & 1.000 & 0.104 \\
\hline Management of working environment & $83.9 \% \pm 19.6$ & $83.8 \% \pm 21.8$ & 0.999 & 0.007 \\
\hline
\end{tabular}

Data are presented as mean + standard deviation in \%. p = significance, $\mathrm{d}=$ Cohen's d (educational effect size). 
Table 4a. Point in Time 2: Performance results for technical skills for sterile working during preparing a venous access port for medication administration

\begin{tabular}{l|l|l|l|l}
\hline & Oral Feedback & Video Feedback & $\mathrm{p}$ & $\mathrm{d}$ \\
\hline Overall score & $52.2 \% \pm 20.8$ & $45.3 \% \pm 19.0$ & 0.078 & 0.345 \\
\hline Personal preparation & $63.2 \% \pm 34.2$ & $62.5 \% \pm 32.9$ & 0.996 & 0.021 \\
\hline Sterile opening and preparation of material & $63.8 \% \pm 25.0$ & $65.2 \% \pm 24.7$ & 0.983 & 0.055 \\
\hline Palpation of port, skin desinfection & $51.4 \% \pm 31.6$ & $47.7 \% \pm 30.8$ & 0.526 & 0.120 \\
\hline Donning of sterile gloves & $67.6 \% \pm 26.0$ & $63.6 \% \pm 29.2$ & 0.774 & 0.146 \\
\hline Application of sterile adhesive drape & $50.0 \% \pm 44.4$ & $30.3 \% \pm 41.3$ & 0.109 & 0.459 \\
\hline Piercing port with needle & $35.3 \% \pm 30.2$ & $23.9 \% \pm 27.2$ & 0.261 & 0.399 \\
\hline
\end{tabular}

Data are presented as mean + standard deviation in \%. $\mathrm{p}=$ significance, $\mathrm{d}=$ Cohen's $\mathrm{d}$ (educational effect size).

Table 4b. Point in Time 2: Performance results for non-technical skills for sterile working during preparing a venous access port for medication administration

\begin{tabular}{l|l|l|l|l}
\hline & Oral Feedback & Video Feedback & $\mathrm{p}$ & $\mathrm{d}$ \\
\hline Overall score & $60.9 \% \pm 20.0$ & $63.6 \% \pm 22.0$ & 0.483 & 0.129 \\
\hline Introduction to the patient & $90.2 \% \pm 28.7$ & $85.9 \% \pm 31.5$ & 0.999 & 0.141 \\
\hline Articulation/ terminology & $75.1 \% \pm 26.0$ & $76.7 \% \pm 25.1$ & 0.959 & 0.062 \\
\hline Structure of working & $58.5 \% \pm 23.2$ & $65.2 \% \pm 25.5$ & 0.652 & 0.275 \\
\hline Behavior/professional attire and appearance & $58.5 \% \pm 22.5$ & $63.3 \% \pm 26.0$ & 0.345 & 0.199 \\
\hline Patient handling & $60.8 \% \pm 21.5$ & $63.7 \% \pm 24.3$ & 0.987 & 0.129 \\
\hline Manangement of working environment & $22.3 \% \pm 17.4$ & $26.7 \% \pm 24.0$ & 0.999 & 0.211 \\
\hline
\end{tabular}

Data are presented as mean + standard deviation in \%. p = significance, $\mathrm{d}=$ Cohen's d (educational effect size). 